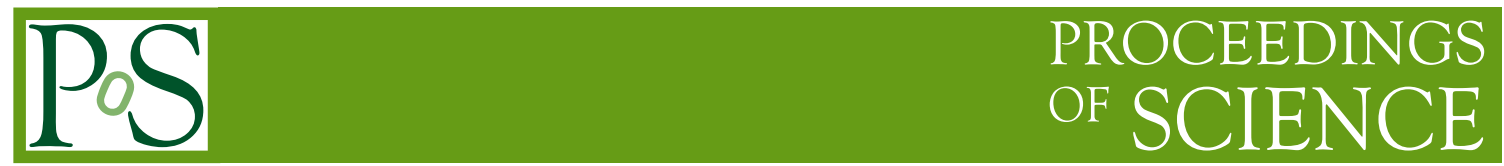

\title{
Status of the MUonE experiment
}

\author{
Giovanni Abbiendi ${ }^{1, *}$ \\ INFN - Sezione di Bologna, \\ Viale C. Berti Pichat, 6/2, 40127 Bologna - Italy \\ E-mail: giovanni.abbiendi@cern.ch
}

The MUonE experiment aims at an independent and very precise determination of the leading hadronic contribution to the muon magnetic moment, based on an alternative method, complementary to the existing ones. This can be achieved by measuring with unprecedented precision the shape of the differential cross section of $\mu e$ elastic scattering, using the intense muon beam available at CERN, with energy of $150 \mathrm{GeV}$, off atomic electrons of a light target. The status of the project is presented, with recent results in preparation for the test run scheduled in 2021 with a reduced detector.

40th International Conference on High Energy physics - ICHEP2020

July 28 - August 6, 2020

Prague, Czech Republic (virtual meeting)

\footnotetext{
${ }^{1}$ For the MUonE collaboration.

* Speaker
} 


\section{Introduction}

The muon magnetic moment is one of the most precisely measured quantities (better than one part per billion). It is also calculable with extremely high precision in the Standard Model, hence it constitutes one of the most stringent tests of the theory. Currently the reference measurement [1] shows a significant discrepancy of $3.7 \sigma$ with respect to the Standard Model prediction [2], which is one of very few deviations in the high energy physics landscape. A new measurement is underway at Fermilab [3], targeting a reduction of the experimental error by a factor of 4, and another one is planned at J-PARC [4]. In view of the forthcoming improved measurements a reduction of the theoretical error is also desirable, in particular of its largest contribution, related to the hadronic vacuum polarization, which is not calculable in perturbation theory. At leading order this is usually evaluated via a dispersion integral of the hadron production cross section in $e^{+} e^{-}$annihilation [5]. The low energy region, with the many resonances and threshold effects, is enhanced in the integral, and constitutes the main difficulty of the method. Alternative evaluations by lattice QCD are continuously progressing, and are expected to become more and more competitive in the near future [2].

A novel approach has been proposed in [6], to determine the leading hadronic contribution

$a_{\mu}^{\mathrm{HLO}}$ from a measurement of the effective electromagnetic coupling in the space-like region, where the vacuum polarization is a smooth function. Its master equation is:

$$
a_{\mu}^{\mathrm{HLO}}=\frac{\alpha}{\pi} \int_{0}^{1} d x(1-x) \Delta \alpha_{\mathrm{had}}[t(x)],
$$

where $\Delta \alpha_{\text {had }}(t)$ is the hadronic contribution to the running of the QED coupling, evaluated at

$$
t(x)=-\frac{x^{2} m_{\mu}^{2}}{1-x}<0,
$$

the space-like (negative) squared four-momentum transfer. By measuring the running of the effective coupling:

$$
\alpha(t)=\frac{\alpha(0)}{1-\Delta \alpha(t)},
$$

where $\alpha(0)=\alpha$ is the fine-structure constant, the hadronic contribution $\Delta \alpha_{\text {had }}(t)$ can be extracted by subtracting from $\Delta \alpha(t)$ the purely leptonic part $\Delta \alpha_{\text {lep }}(t)$, which can be calculated to very high precision in QED.

To date there exist very few direct measurements of the running of $\alpha$ in the space-like region, the most precise one was obtained by the OPAL experiment [7], from small-angle Bhabha scattering, and reached the sensitivity for the observation of the hadronic contribution.

Recently the MUonE experiment [8] has been proposed, to measure the hadronic running of $\alpha(t)$ from $\mu e$ elastic scattering at low energy. Here we will report the status of the project and few recent results.

\section{MUonE experimental proposal}

MUonE aims at a very precise measurement of the shape of the differential cross section of $\mu e$ elastic scattering, with the CERN M2 muon beam $\left(E_{\mu} \sim 150 \mathrm{GeV}\right)$ off atomic electrons of a light Be 
or $\mathrm{C}$ target. The hadronic contribution $\Delta \alpha_{\text {had }}(t)$ has a tiny variation across the probed kinematical range $0<x<0.932$ (equivalent to $0<-t<0.143 \mathrm{GeV}^{2}$ ), changing from a vanishingly small value at low $x$ to about $10^{-3}$ at the peak of the integrand in Eq. 1, which occurs at $x=0.914$ $\left(t=-0.108 \mathrm{GeV}^{2}\right)$. A competitive determination of $a_{\mu}^{\mathrm{HLO}}$ requires a precision of $O\left(10^{-2}\right)$ in the measurement of the hadronic running, which translates into an unprecedented precision of $O\left(10^{-5}\right)$ in the shape of the differential cross section. To reach this impressive accuracy a modular detector is proposed, consisting of a sequence of detection stations, each one made of a passive element serving as target (1.5 cm Be plate) and active planes made of silicon microstrip detectors for tracking, with length of 1 meter and transverse dimensions of about $10 \mathrm{~cm}$. The current design of a MUonE tracking station is shown in Fig. 1. The complete layout foresees an array of 40 such stations, followed by an electromagnetic calorimeter (ECAL) and a muon detector at the end. This design could reach the target statistical sensitivity in three years running time at the M2 beam, with an integrated luminosity of $1.5 \times 10^{7} \mathrm{nb}^{-1}$. Clearly the ultimate challenge is keeping the systematics at the same level as the statistical accuracy.

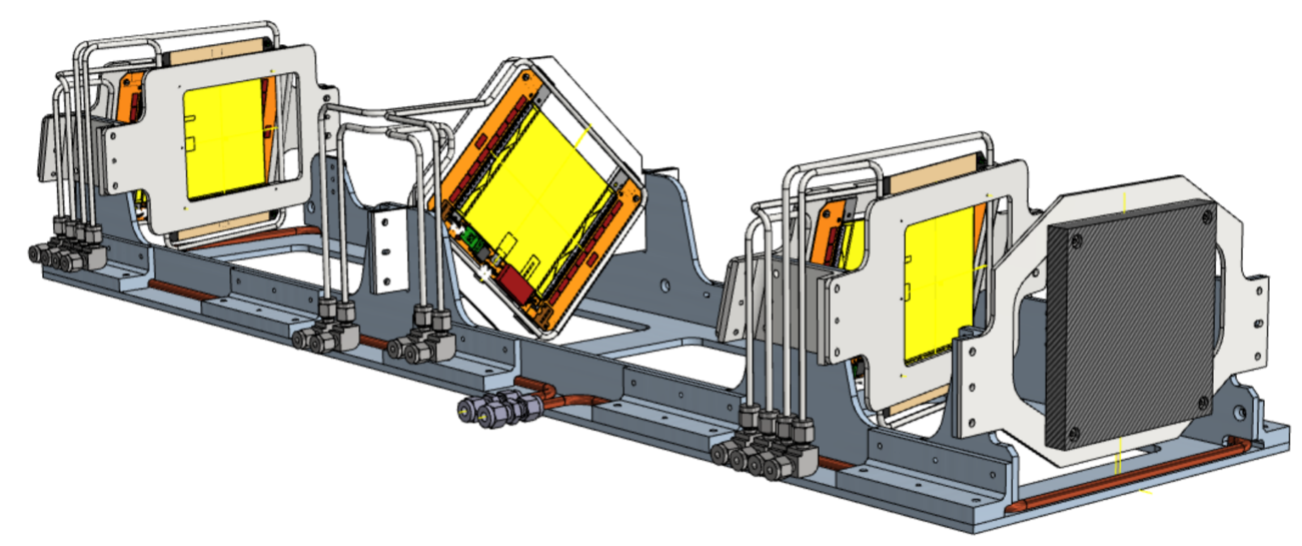

Figure 1: CAD drawing of a MUonE tracking station.

The basic tracking unit has been chosen to be the $2 \mathrm{~S}$ module developed for the upgrade of the CMS outer tracker [10]. It consists of two close-by planes of silicon microstrips, providing track triggering capability at $40 \mathrm{MHz}$, with inherent suppression of background from single-layer hits or large-angle tracks. It has a large active area of about $10 \times 10 \mathrm{~cm}^{2}$, allowing to completely cover the relevant MUonE angular acceptance with a single module, thus assuring the best uniformity. The position resolution $\sim 20 \mu \mathrm{m}$ is adequate and can be further improved to $\sim 10 \mu \mathrm{m}$ with a $\sim 15^{\circ}$ tilt around the strip axis. With respect to the LHC operation the main difference for MUonE will be the asynchronous nature of the signals from the $\mu e$ scattering events. In principle this can be managed with a specific configuration of the front-ends but is a crucial issue which will need thorough tests.

The MUonE project has been submitted to the CERN SPS Committee in June 2019 [9], obtaining positive recommendations and requests for the necessary future milestones. 


\section{Test Run 2021}

A first important milestone is a three-week Test Run allocated in the last part of 2021 at the CERN M2 beam line, with full intensity muon beam. The selected location is upstream of the COMPASS detector, after its Beam Momentum Spectrometer (BMS). The MUonE setup will consist of two tracking stations followed by the ECAL, with an additional station (without target) upstream to track the incoming muons.

Each tracking station is a 1-meter long structure (Fig. 1), with a target plate followed by three equally-spaced XY supermodules, each one made of two $2 \mathrm{~S}$ modules with orthogonal strips. The first and third supermodules, measuring $\mathrm{X}$ and $\mathrm{Y}$ transverse coordinates, have tilted detectors by $\sim 15^{\circ}$ on the two orthogonal planes, to improve the hit position resolution. The middle supermodule is rotated by $45^{\circ}$ around the $z$ axis to solve the reconstruction ambiguities. There are stringent requests on the mechanical stability of the tracking stations, which has to be better than $10 \mu \mathrm{m}$, in particular on the longitudinal size. Therefore the support structure is made of Invar (Fe-Ni alloy), which has a very low coefficient of thermal expansion, is easy to machine and relatively cheap. A cooling system is also designed, in addition to an enclosure to stabilize the room temperature within $1^{o}-2^{o}$.

The purpose of this Test Run is crucial. It should confirm the system engineering, test the mechanical and thermal stability, the procedure for the alignment (hardware and software), the readout chain and the trigger strategy to identify and reconstruct $\mu e$ events.

Assuming to achieve these prime objectives and to have enough time available for running, we have tried to assess the physics potential of the chosen setup in the best-case scenario. Considering the standard SPS efficiency and full beam intensity, the two stations could potentially yield $\sim 1 \mathrm{pb}^{-1} /$ day. By allowing for a reasonable time for detector commissioning and considering possible DAQ inefficiencies we could in principle integrate up to $\sim 5 \mathrm{pb}^{-1}$ of good data during a first physics run, corresponding to $\sim 10^{9} \mu e$ scattering events with electron energy greater than $1 \mathrm{GeV}$. Such a data sample would have enough sensitivity to measure the leptonic running of $\alpha$ and, allowing some optimism, could even give initial sensitivity to the hadronic running.

The hadronic contribution to the running of $\alpha$ is most easily displayed by considering the ratio $R_{\text {had }}$ of the observed angular distributions with the theoretical predictions evaluated for $\alpha(t)$ corresponding to only the leptonic running. Fig. 2-left shows the expectation for the muon angular distribution, obtained from a NLO MC generator [11] plus a fast simulation of multiple Coulomb scattering (MCS) and the detector intrinsic resolution. The extraction of the hadronic contribution is carried out by a template fit method [9], which here is simplified in a 1D fit as the limited statistics allows to determine just a linear deviation on the shape. The fit gives $K=0.136 \pm 0.026$ as the slope of the observable hadronic running at the purely statistical level.

It is important to assess the expected systematic errors, which will have to be fully understood in the Test Run conditions to estimate their impact in the full experiment. One of the most important systematics is related to the estimated MCS, affecting in particular the low energy electrons. This has been also studied in a dedicated beam test in 2017 [12]. Fig. 2-right shows the effect on the muon angular distribution of a flat $\pm 1 \%$ error on the MCS core width. After a selection cut $\theta_{e}<20 \mathrm{mrad}$ on the electron angle and by restricting the fit region to $\theta_{\mu}>0.4 \mathrm{mrad}$ one obtains: $K=0.137 \pm 0.033$ (stat) ${ }_{-0.004}^{+0.006}$ (syst). 

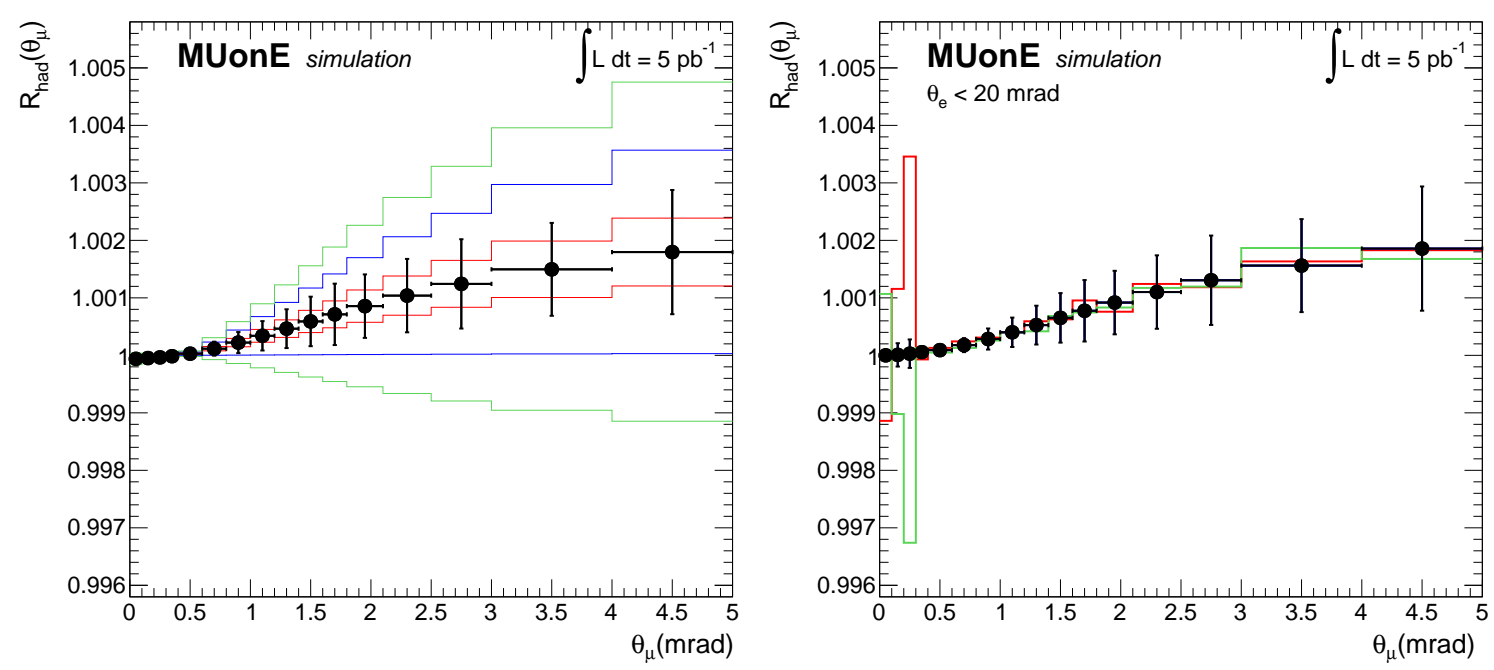

Figure 2: Ratio $R_{\text {had }}$ of the expected muon angular distribution and the prediction obtained with only leptonic running in $\alpha(t)$. The error bars correspond to the statistical uncertainties for an integrated luminosity of $5 \mathrm{pb}^{-1}$ assumed for the 2021 Test Run. Left: The histograms show the templates for few values of the slope K. Right: The effect of a $+1 \%(-1 \%)$ systematic error on the assumed core width of the MCS distribution is shown by the red (green) histogram. A cut $\theta_{e}<20 \mathrm{mrad}$ is applied here to reduce the impact of this systematic.

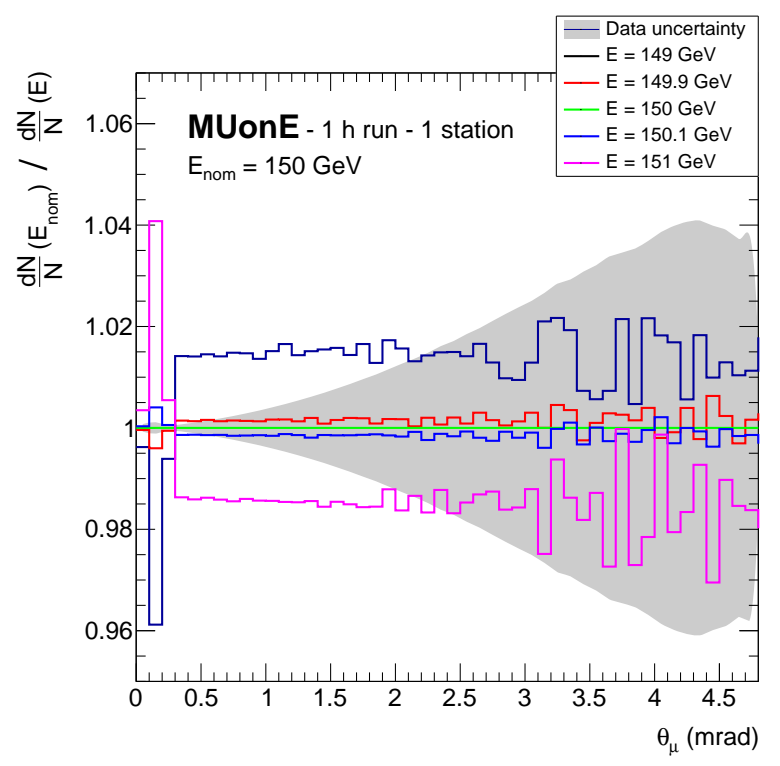

Figure 3: Effect of a shift of $\pm(0.1-1.0) \mathrm{GeV}$ in the average beam energy with respect to the nominal value $E_{\text {nom }}=150 \mathrm{GeV}$. The histograms show the expected distortions on the muon angular distribution, obtained from MC samples with variable energy. The grey band represents the statistical uncertainty corresponding to the expected data collected in one hour running time by one station. 
Another crucial systematic effect is related to the knowledge of the average beam energy scale. This is known from the accelerator at a level of about $1 \%$. The BMS spectrometer can measure individual incoming muons with $0.8 \%$ resolution, and given the high muon beam intensity it can provide an excellent monitor of time variations of the average scale. However it cannot assess the systematic uncertainty of the average energy scale, which has to be controlled by a physical process. The kinematics of the elastic $\mu e$ scattering has been identified as the useful method [9], in particular the average angle of the two outgoing tracks, which does not need $\mu-e$ identification. For illustration purpose Fig. 3 shows the effect on the muon angular distribution of a systematic error of $\pm(0.1-1.0) \mathrm{GeV}$ on the assumed average beam energy. The expected distortion is compared to the statistical uncertainty corresponding to one hour running time in one station. It is clear that the energy calibration by the kinematical method would already outperform the precision of the scale obtained from the accelerator. The beam energy scale will be calibrated on each tracking station independently, aiming at an ultimate precision for the final detector better than $3 \mathrm{MeV}$ in less than one week of run.

\section{Theoretical progress}

The MUonE challenging goal of a precision determination of $a_{\mu}^{\mathrm{HLO}}$ depends also crucially on the availability of very precise theoretical calculations for $\mu e$ scattering. The full set of NLO QED and electroweak corrections was computed with the development of a fully exclusive MC event generator [11]. The NNLO hadronic corrections have been recently computed in [13, 14]. The full NNLO QED corrections are not yet available, although several important steps were taken in [15-17]. For a state-of-the-art review see [18] and references therein. On top of that a further milestone was recently achieved by the development of two independent fully exclusive MC codes, featuring the exact NNLO photonic corrections on the leptonic legs, including all mass terms $[19,20]$. Their results have been compared and are in very good agreement. Resummation of leading terms in higher orders (Parton shower and YFS) matched to (N)NLO will be also necessary and is underway. Possible new physics effects in muon-electron collisions are expected to lie below MUonE sensitivity [21, 22].

\section{Next steps}

The MUonE Collaboration currently includes groups from CERN, China, Germany, Greece, Italy, Poland, Russia, Switzerland, UK, and USA, in addition to involved theorists from other countries.

On the experimental side an intense activity is going on for the preparation of the Test Run, scheduled for the end of 2021. This will be a proof of concept for the overall project and is expected to accurately evaluate the experimental systematics, in order to assess the potential of the proposed detector design to yield a competitive measurement. If successful, a full proposal will be prepared including support from the results of this run. The full detector construction could then take place starting from 2022 with the prospect of a substantial running time during the LHC Run3. 


\section{References}

[1] G. W. Bennett et al. [Muon g-2 Collaboration], Final Report of the Muon E821 Anomalous Magnetic Moment Measurement at BNL, Phys. Rev. D 73 (2006) 072003 [hep-ex/0602035].

[2] T. Aoyama et al., The anomalous magnetic moment of the muon in the Standard Model, Phys. Rept. 887 (2020) 1 [hep-ph/2006. 04822].

[3] J. Grange et al. [Muon g-2 Collaboration], Muon (g-2) Technical Design Report, arXiv:1501.06858 [physics.ins-det].

[4] M. Abe et al., A New Approach for Measuring the Muon Anomalous Magnetic Moment and Electric Dipole Moment, PTEP 2019 (2019) 053C02 [physics .ins-det/1901.03047].

[5] M. Davier, A. Hoecker, B. Malaescu and Z. Zhang, A new evaluation of the hadronic vacuum polarisation contributions to the muon anomalous magnetic moment and to $\boldsymbol{\alpha}\left(\mathbf{m}_{\mathbf{Z}}^{\mathbf{2}}\right)$, Eur. Phys. J. C 80 (2020) 241 [erratum: Eur. Phys. J. C 80 (2020) 410 ] [hep-ph/1908.00921].

[6] C. M. Carloni Calame, M. Passera, L. Trentadue and G. Venanzoni, A new approach to evaluate the leading hadronic corrections to the muon g-2, Phys. Lett. B 746 (2015) 325 [hep-ph/1504.02228].

[7] G. Abbiendi et al. [OPAL Collaboration], Measurement of the running of the QED coupling in small-angle Bhabha scattering at LEP, Eur. Phys. J. C 45 (2006) 1 [hep-ex/0505072].

[8] G. Abbiendi et al., Measuring the leading hadronic contribution to the muon g-2 via $\mu$ e scattering, Eur. Phys. J. C 77 (2017) 139 [hep-ex/1609.08987].

[9] G. Abbiendi et al. [MUonE Collaboration], Letter of Intent: the MUonE project, CERNSPSC-2019-026 / SPSC-I-252

[10] CMS Collaboration, "The Phase-2 Upgrade of the CMS Tracker", CERN-LHCC-2017-009 / CMS-TDR-014

[11] M. Alacevich, C. M. Carloni Calame, M. Chiesa, G. Montagna, O. Nicrosini and F. Piccinini, Muon-electron scattering at NLO, JHEP 02 (2019) 155 [hep-ph/1811.06743].

[12] G. Abbiendi et al., Results on multiple Coulomb scattering from 12 and $20 \mathrm{GeV}$ electrons on carbon targets, JINST 15 (2020) P01017 [physics.ins-det/1905 .11677].

[13] M. Fael, Hadronic corrections to $\mu$-e scattering at NNLO with space-like data, JHEP 02 (2019), 027 [hep-ph/1808. 08233].

[14] M. Fael and M. Passera, Muon-Electron Scattering at Next-To-Next-To-Leading Order: The Hadronic Corrections, Phys. Rev. Lett. 122 (2019) 192001 [hep-ph/1901.03106].

[15] P. Mastrolia, M. Passera, A. Primo and U. Schubert, Master integrals for the NNLO virtual corrections to $\mu$ e scattering in QED: the planar graphs, JHEP 11 (2017) 198 [hep-ph/1709. 07435]. 
[16] S. Di Vita, S. Laporta, P. Mastrolia, A. Primo and U. Schubert, Master integrals for the NNLO virtual corrections to $\mu$ scattering in QED: the non-planar graphs, JHEP 09 (2018) 016 [hep-ph/1806.08241].

[17] S. Di Vita, T. Gehrmann, S. Laporta, P. Mastrolia, A. Primo and U. Schubert, Master integrals for the NNLO virtual corrections to $q \bar{q} \rightarrow t \bar{t}$ scattering in $Q C D$ : the non-planar graphs, JHEP 06 (2019) 117 [hep-ph/1904 . 10964].

[18] P. Banerjee et al., Theory for muon-electron scattering @ 10 ppm: A report of the MUonE theory initiative, Eur. Phys. J. C 80 (2020) 591 [hep-ph/2004 . 13663].

[19] C. M. Carloni Calame, M. Chiesa, S. M. Hasan, G. Montagna, O. Nicrosini and F. Piccinini, Towards muon-electron scattering at NNLO, JHEP 11 (2020) 028 [hep-ph/2007. 01586].

[20] P. Banerjee, T. Engel, A. Signer and Y. Ulrich, QED at NNLO with McMule, SciPost Phys. 9 (2020) 027 [hep-ph/2007.01654].

[21] P. S. B. Dev, W. Rodejohann, X. J. Xu and Y. Zhang, MUonE sensitivity to new physics explanations of the muon anomalous magnetic moment, JHEP 05 (2020) 053 [hep-ph/2002 . 04822].

[22] A. Masiero, P. Paradisi and M. Passera, New physics at the MUonE experiment at CERN, Phys. Rev. D 102 (2020) 075013 [hep-ph/2002 .05418]. 